\title{
Long-Term Efficacy of a Treat-and-Extend Regimen with Ranibizumab in Patients with Neovascular Age-Related Macular Disease: An Open-Label 12-Month Extension to the CANTREAT Study
}

\author{
Peter J. Kertes ${ }^{a}$ Tom Sheidow ${ }^{b} \quad$ Geoff Williams $^{c} \quad$ Mark Greve $^{d} \quad$ Ivan J. Galic ${ }^{e}$ \\ Jason Baker ${ }^{f}$ on behalf of the CANTREAT Investigators

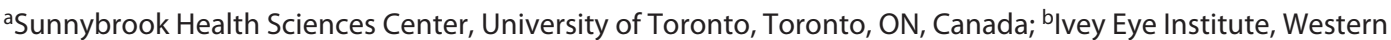 \\ University, London, ON, Canada; ' Calgary Retina Consultants, University of Calgary, Calgary, AB, Canada; ${ }^{\mathrm{d}}$ Alberta \\ Retina Consultants, University of Alberta, Edmonton, AB, Canada; 'Institut de la Rétine de Montréal, Montréal, QC, \\ Canada; ${ }^{\mathrm{f}}$ Novartis Pharmaceuticals Canada, Dorval, QC, Canada
}

\section{Keywords}

Neovascular age-related macular degeneration - Treat and extend $\cdot$ Best-corrected visual acuity $\cdot$ Injections

\begin{abstract}
Introduction: The objective of this study is to assess the long-term effectiveness of a treat-and-extend (T\&E) anti-vascular endothelial growth factor regimen in patients with neovascular age-related macular degeneration who remain on T\&E and those switched from once-monthly (OM) dosing to T\&E (OM-T\&E). Methods: In this 12-month extension of the 2-year CANTREAT study, patients received intravitreal ranibizumab $0.5 \mathrm{mg}$ in a T\&E regimen. Main outcome measures included mean change in best-corrected visual acuity (BCVA) from baseline and from month 24 to month 36; percentages of patients who gained $\geq 5, \geq 10$, or $\geq 15$ Early Treatment of Diabetic Retinopathy Study (ETDRS) letters or lost $\geq 5, \geq 10$, or $\geq 15$ letters from baseline and from month 24 to month 36; and number of injections administered from baseline and from month 24 to month 36 for both groups. Results: Of the 139 patients (73 T\&E, 66 OM-T\&E) in the extension, 121 (68
\end{abstract}

T\&E, 53 OM-T\&E) completed 36 months. Mean (standard deviation [SD]) BCVA changes from baseline to the extension last visit (month 33-36) were +6.6 (11.4) letters in the T\&E group and +4.8 (14.3) letters in the OM-T\&E group, representing maintenance of 24-month gains. The mean (SD) numbers of injections during the extension were 7.3 (2.7) for T\&E and 7.1 (2.8) for OM-T\&E. Discussion/Conclusion: These findings suggest that after 36 months of treatment, the mean BCVA improvement achieved at 24 months is maintained for both the patients exclusively treated with the T\&E regimen and those that switched to T\&E after 24 months in the OM regimen.

(C) 2021 The Author(s). Published by S. Karger AG, Basel

Meeting Presentation Presented at: Annual Meeting of the American Academy of Ophthalmology, October 12-15, 2019; 2020 Canadian Retina Society Meeting, February 13-16, 2020, Banff, Alberta; Association for Research in Vision in Ophthalmology 2020 Annual Meeting, May 3-7, 2020 (virtual); 2020 Canadian Ophthalmological Society Annual Meeting and Exhibition, June 25-28, 2020 (virtual); 38th Annual Scientific Meeting of the American Society of Retina Specialists, July 24-26, 2020 (virtual). karger@karger.com www.karger.com/oph

Karger $\stackrel{\text { ' }}{5}$

BOPEN ACCESS
(C) 2021 The Author(s)

Published by S. Karger AG, Basel

This is an Open Access article licensed under the Creative Common Attribution-NonCommercial-4.0 International License (CC BY-NC) (http://www.karger.com/Services/OpenAccessLicense), applicable to the online version of the article only. Usage and distribution for commercial purposes requires written permission.
Correspondence to:

Peter J. Kertes, peter.kertes@ sunnybrook.ca 


\section{Introduction}

Neovascular age-related macular degeneration (nAMD) is among the most common causes of severe and irreversible vision loss, particularly in developed countries [1-3]. nAMD increases significantly in prevalence and severity with advancing age [3-6]. If left untreated, patients with nAMD can expect to experience profound vision loss.

Ongoing treatment and monitoring are essential among nAMD patients to preserve vision and reduce the risk and severity of progression. Results from long-term anti-vascular endothelial growth factor (anti-VEGF) studies such as the Seven-Year Observational Update of Macular Degeneration Patients Post-MARINA/ANCHOR and HORIZON Trials (SEVEN-UP) [7], the Comparison of Age-Related Macular Degeneration Treatments Trials (CATT; 5 years) [8], and the TReat and EXtend Protocol in Patients with Wet Age-related Macular Degeneration (TREX-AMD) study (3 years) [9] demonstrated the effectiveness of long-term anti-VEGF therapy in the maintenance of VA among patients with nAMD. Reductions in the number of injections over these time periods, however, were associated with incremental loss of the VA gains originally achieved.

The treatment and monitoring associated with management of nAMD are themselves a source of considerable burden on patients and their caregivers. The main factors associated with the considerable burden to patients are travel and time constraints for caregivers [10, 11 . Burden of nAMD management is also seen across healthcare systems [12], and frequently leads to undertreatment in the real-world management of nAMD [1316]. Reduction of this treatment burden is considered by retina specialists to be the greatest unmet need regarding management of nAMD [17].

To address the multiple challenges associated with the management of patients with nAMD, retina specialists are increasingly using a treat-and-extend (T\&E) strategy, which has been shown in several studies to reduce the number of monitoring visits and anti-VEGF injections while safely and effectively maintaining or improving VA and minimizing nAMD-related disease activity (i.e., retinal fluid and hemorrhage) [18-26]. According to a large meta-analysis, nAMD patients treated with a T\&E strategy appeared to achieve better VA outcomes than asneeded dosing [21].

The multicenter Canadian Treat and Extend Analysis Trial with Ranibizumab (CANTREAT; clinicaltrials.gov identifier: NCT02103738) met its primary endpoint of noninferiority between T\&E and once-monthly (OM) regimens by assessing the mean (standard deviation [SD]) change in best-corrected VA (BCVA) ETDRS letters from baseline to Month 12, (+8.4 [11.9] vs. +6.0 [11.9] letters, respectively; $p=0.017$ ), and a secondary analysis demonstrated significantly fewer injections with $\mathrm{T} \& \mathrm{E}$ versus $\mathrm{OM}$ dosing (9.4 vs. 11.8 injections; mean difference: 2.46 ; $95 \%$ confidence interval [CI], 2.23-2.68; $p<0.001$ ) [27]. These outcomes were supported by the CANTREAT 24-month findings [28]. The changes (SD) in BCVA for the T\&E and OM groups were +6.8 (14.1) letters and +6.0 (12.6) letters, respectively ( $95 \% \mathrm{CI},-1.6$ to $3.3 ; p=0.21$ ), and the average numbers of injections were 17.6 and 23.5 , respectively (mean difference 5.9; 95\% CI, 5.4-6.5; $p<0.001$ ). The objective of this 12-month CANTREAT extension is to assess the long-term effectiveness of a T\&E regimen in patients who remain on $\mathrm{T} \& \mathrm{E}$ as well as those who are switched from OM to T\&E (OM-T\&E).

\section{Materials and Methods}

Study Design

This is a 12-month, open-label extension of the 2-year, prospective, randomized, multicenter, post-authorization CANTREAT study. In the original study, treatment-naïve patients with $\mathrm{nAMD}$ were originally randomized to either OM or T\&E dosing of intravitreal ranibizumab $0.5 \mathrm{mg}$, and they were maintained on these regimens for 24 months. A detailed explanation and description of the protocol and design for CANTREAT were reported previously [27]. The study and written informed consent form for patients participating in the 12-month extension phase were approved by each site's institutional review board, independent Ethics Committee, or research ethics board. As with the initial CANTREAT study, this extension was conducted in adherence to the tenets of the Declaration of Helsinki [29]. All patients provided written informed consent prior to enrollment in this extension.

The patients invited to participate in the 12-month extension had completed the 24-month CANTREAT study but had not yet completed the 24-month follow-up, when the 12-month extension (version date May 1, 2017) was finalized and ready to be executed. No additional selection criteria were applied for inclusion. Participants consented to receive $0.5-\mathrm{mg}$ injections of ranibizumab under a T\&E regimen for an additional 12 months. Patients were assessed according to their original treatment arm: those originally assigned to a T\&E regimen continued on T\&E and those originally assigned to an OM regimen switched to T\&E (OM-T\&E) for the 12-month extension period. Attempts to extend treatment intervals and responses to disease instability during the extension were performed as per the original CANTREAT protocol (online suppl. Fig. S1; see www.karger.com/doi/10.1159/000521517 for all online suppl. material).

\section{Outcome Measures}

The exploratory objectives of the CANTREAT 12-month extension were to describe the effectiveness of the T\&E dosing regi- 


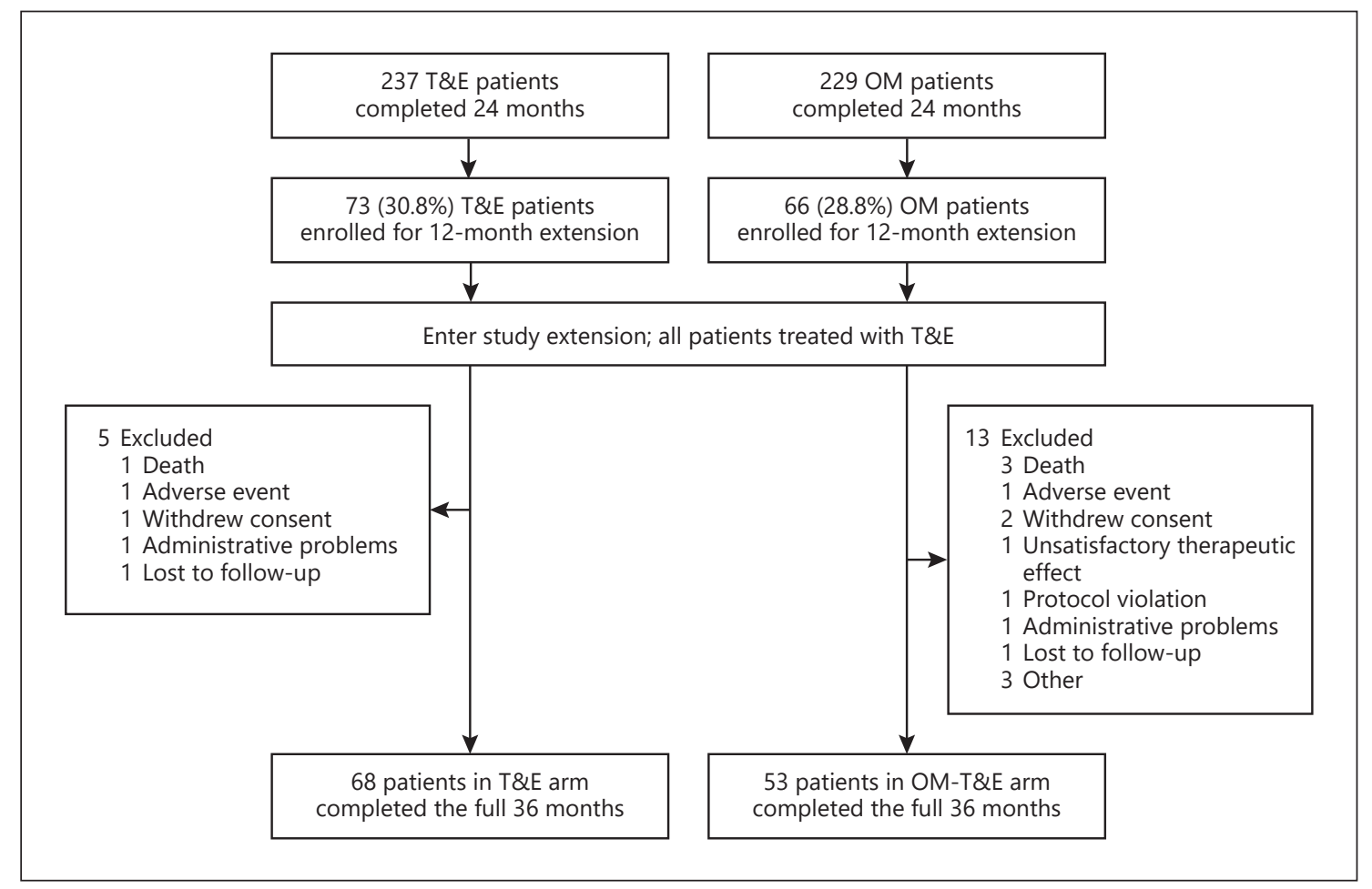

Fig. 1. Flow of study participants.

men at month 36 by treatment arm, to describe the effectiveness of switching from an OM regimen to a T\&E regimen upon completion of the 24-month study, and to compare the effectiveness of T\&E from months 24 to 36 between patients in the 2 treatment arms. The variables related to the exploratory efficacy objectives included: the mean changes in BCVA from baseline to month 36 and from months 24 to 36; percentages of patients with gains of $\geq 5, \geq 10$, or $\geq 15$ letters, or with losses of $\geq 5, \geq 10$, or $\geq 15$ letters from baseline to month 36 , and the number of injections administered from baseline to month 36 and from months 24 to 36, all by treatment arm. Month 36 is defined as the value obtained at the week 156 visit, which may vary in time due to the individualized nature of the T\&E regimen. For accuracy of comparison, mean baseline, 12-, and 24-month outcomes were determined for the subpopulation who participated in the extension and are reflected in the results.

An ophthalmic examination, evaluation of BCVA, and optical coherence tomography (OCT) were performed at all injection visits and at 160-week follow-up. Ophthalmic examination included at least a slit-lamp examination, fundus examination, and tonometry. Fluorescein angiography and fundus autofluorescence (subset of sites) were performed at 104 weeks (first treatment visit) and 156 weeks (last treatment visit).

\section{Statistical Analysis}

A sample of convenience was used in which patients completing the 24-month study were offered to participate in the 12-month extension. The primary efficacy analysis focused on change from baseline in BCVA using a repeated measures model, focusing on months 12, 24, and 36. Change from month 3 was also included as this was the end of the titration period, as well as change from months 12 and 24. The model also incorporated baseline BCVA and baseline OCT (as measured by central retinal thickness [CRT] levels) as covariates. If the repeated measures analysis was significant, individual $t$ tests were performed by visit $(12,24$, and 36 months) versus baseline. Student's $t$ test for independent samples was used to assess the unadjusted statistical significance of the between-group difference for these comparisons. Estimates were presented with the appropriate $95 \%$ CIs. Nonparametric testing (if applicable) was used if data were found to deviate significantly from the normal distribution.

All exploratory analyses pertained to the extension phase of this study. Additional subgroup analyses were conducted and included baseline BCVA values (e.g., $<70$ or $\geq 70$, median), intervals of injections for T\&E patients (last injection interval, maximum injection interval), CRT median values, presence of intraretinal fluid (IRF)/ cyst, and presence of subretinal fluid (SRF). All analyses were descriptive based on nonmissing data. T\&E patients were also presented separately with respect to BCVA by CRT for last and maximum injection intervals, respectively. Missing data were maintained as missing unless specified otherwise.

All analyses were conducted using Statistical Analysis Software (SAS) 9.4. Data analysis was performed between August 1 and September 24, 2019. 
Table 1. Demographic and baseline characteristics for extension patients

\begin{tabular}{llll}
\hline Parameter & T\&E $(n=73)$ & OM-T\&E $(n=66)$ & Total $(N=139)$ \\
\hline Mean age (SD), years & $79.0(7.7)$ & $79.1(7.3)$ & $79.1(7.5)$ \\
Female, $n$ (\%) & $44(60.3)$ & $45(68.2)$ & $89(64.0)$ \\
Caucasian/white, $n$ (\%) & $71(97.3)$ & $63(95.5)$ & $134(96.4)$ \\
Mean baseline BCVA in study eye (SD), ETDRS letters & $63.4(10.4)$ & $61.8(12.4)$ & $62.7(11.4)$ \\
Mean CRT of study eye (SD), $\mu \mathrm{mm}$ & $367.6(103.3)$ & $353.2(103.2)$ & $360.8(103.1)$ \\
\hline
\end{tabular}

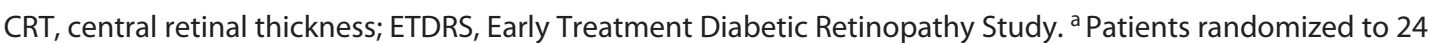
months of T\&E treatment in the main study followed by 12-month T\&E treatment in the extension phase. ${ }^{\mathrm{b}}$ Patients randomized to 24-months of OM treatment in the main study followed by 12 -month T\&E treatment in the extension phase.

Fig. 2. Mean BCVA over time. Data for the Extension Last Visit cohorts are taken from the last visits for the subjects in the study within a window of months 33-36. ETDRS, Early Treatment Diabetic Retinopathy Study; $n$, number of patients with data.

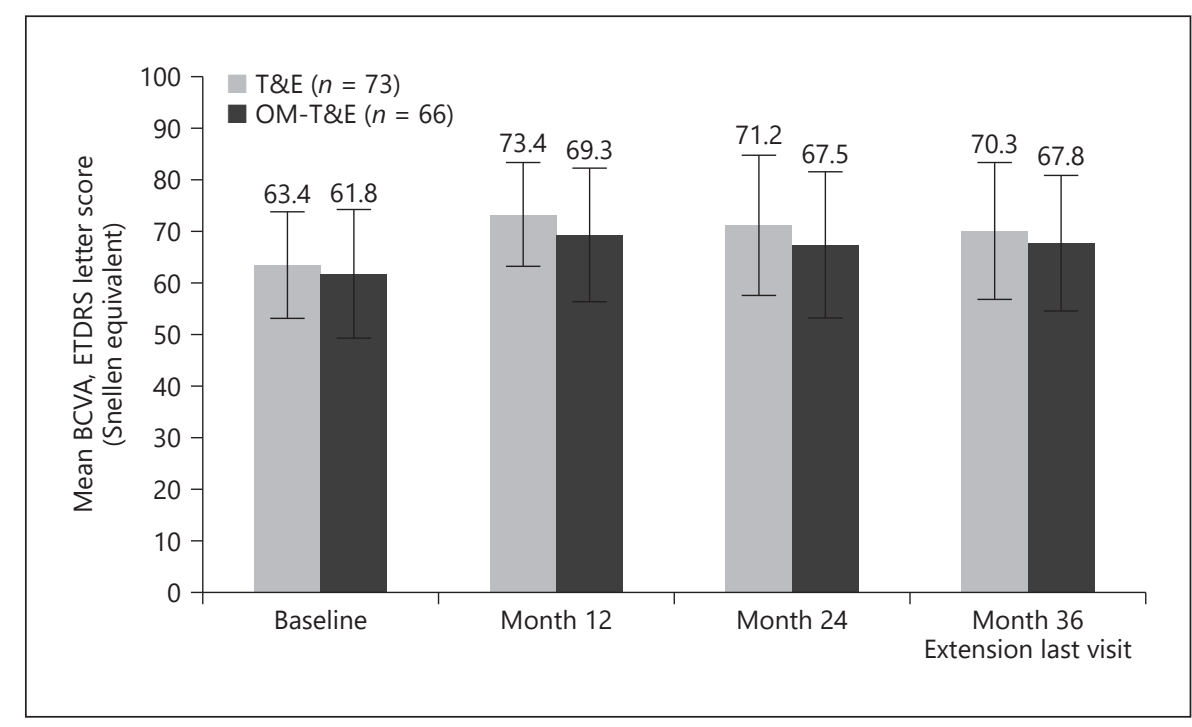

\section{Results}

Of the 139 CANTREAT patients who agreed to participate in the extension (73 T\&E, 66 OM-T\&E), 121 completed the full 36 months (68 T\&E, 53 OM-T\&E). Eighteen patients (12.9\%) discontinued treatment before completing the full 12 months of therapy (Fig. 1). Death was the most common reason for discontinuation (4 patients [2.9\%]); however, there was a relatively even distribution among several factors (online suppl. Table S1). Discontinuations were more common among patients in the OM-T\&E group (13 patients [19.7\%]) versus those who continued with T\&E (5 patients [6.8\%]).

The demographic and baseline characteristics were similar between the treatment groups (Table 1). Mean age (79.1 years), race (96.4\% Caucasian/white), and sex
(64.0\% female) for the patients in the extension are comparable with those of the total initial CANTREAT cohort (online suppl. Table S2) [27].

The mean (SD) gains in BCVA from baseline to the patients' Extension Last Visit (month 33-36) were +6.6 (11.4) letters for T\&E patients $(n=71)$ and $+4.8(14.3)$ letters for OM-T\&E patients $(n=59)$ (Fig. 2), which represent changes of -1.1 (8.0) and -0.6 (7.6) letters, respectively, from 24-month outcomes. Slightly more patients in the $T \& E$ group gained $\geq 15, \geq 10$, and $\geq 5$ letters than those in the OM-T\&E group, and percentages were nearly equivalent $(p=1.00)$ for $\geq 15$ - and $\geq 10$-letter gains. Fewer T\&E patients lost $\geq 15, \geq 10$, and $\geq 5$ letters; however, none of these differences were statistically significant (Table 2). 
Table 2. Gains and losses of best-corrected visual acuity from baseline by treatment arm - extension

\begin{tabular}{|c|c|c|c|}
\hline Parameter & $\mathrm{T} \& \mathrm{E}^{\mathrm{a}}(n=71)$ & OM-T\&E $\mathrm{E}^{\mathrm{b}}(n=59)$ & Difference (T\&E - OM-T\&E) \\
\hline \multicolumn{4}{|c|}{ ETDRS letters gained, $\%$ of patients $\left(95 \% \mathrm{Cl}^{\mathrm{d}}\right)$} \\
\hline$\geq 15$ letters & $21.9(13.1-33.1)$ & $21.2(12.1-33.0)$ & $0.7(-13.0$ to 14.4$) ; p=1.00$ \\
\hline$\geq 10$ letters & $35.6(24.7-47.7)$ & $34.8(23.5-47.6)$ & $0.8(-15.1$ to 16.7$) ; p=1.00$ \\
\hline$\geq 5$ letters & $57.5(45.4-69.0)$ & $53.0(40.3-65.4)$ & $4.5(-12.0$ to 21.0$) ; p=0.61$ \\
\hline \multicolumn{4}{|c|}{ ETDRS letters lost, $\%$ of patients $\left(95 \% \mathrm{Cl}^{\mathrm{d}}\right)$} \\
\hline$\geq 15$ letters & $4.1(0.9-11.5)$ & $7.6(2.5-16.8)$ & $-3.5(-11.3$ to 4.4$) ; p=0.48$ \\
\hline$\geq 10$ letters & $8.2(3.1-17.0)$ & $10.6(4.4-20.6)$ & $-2.4(-12.1$ to 7.4$) ; p=0.77$ \\
\hline$\geq 5$ letters & $15.1(7.8-25.4)$ & $19.7(10.9-31.3)$ & $-4.6(-17.3$ to 8.0$) ; p=0.51$ \\
\hline
\end{tabular}

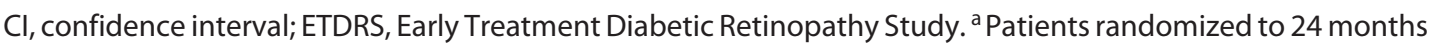
of T\&E treatment in the main study followed by 12 -month T\&E treatment in the extension phase. ${ }^{b}$ Patients randomized to 24-months of OM treatment in the main study followed by 12-month T\&E treatment in the extension phase. ${ }^{\mathrm{C}} \mathrm{Cls}$ for the difference were the asymptotic Wald Cls. ${ }^{d} \mathrm{Cls}$ for treatment arms were calculated based on the binomial distribution for $\geq x x$ letters.

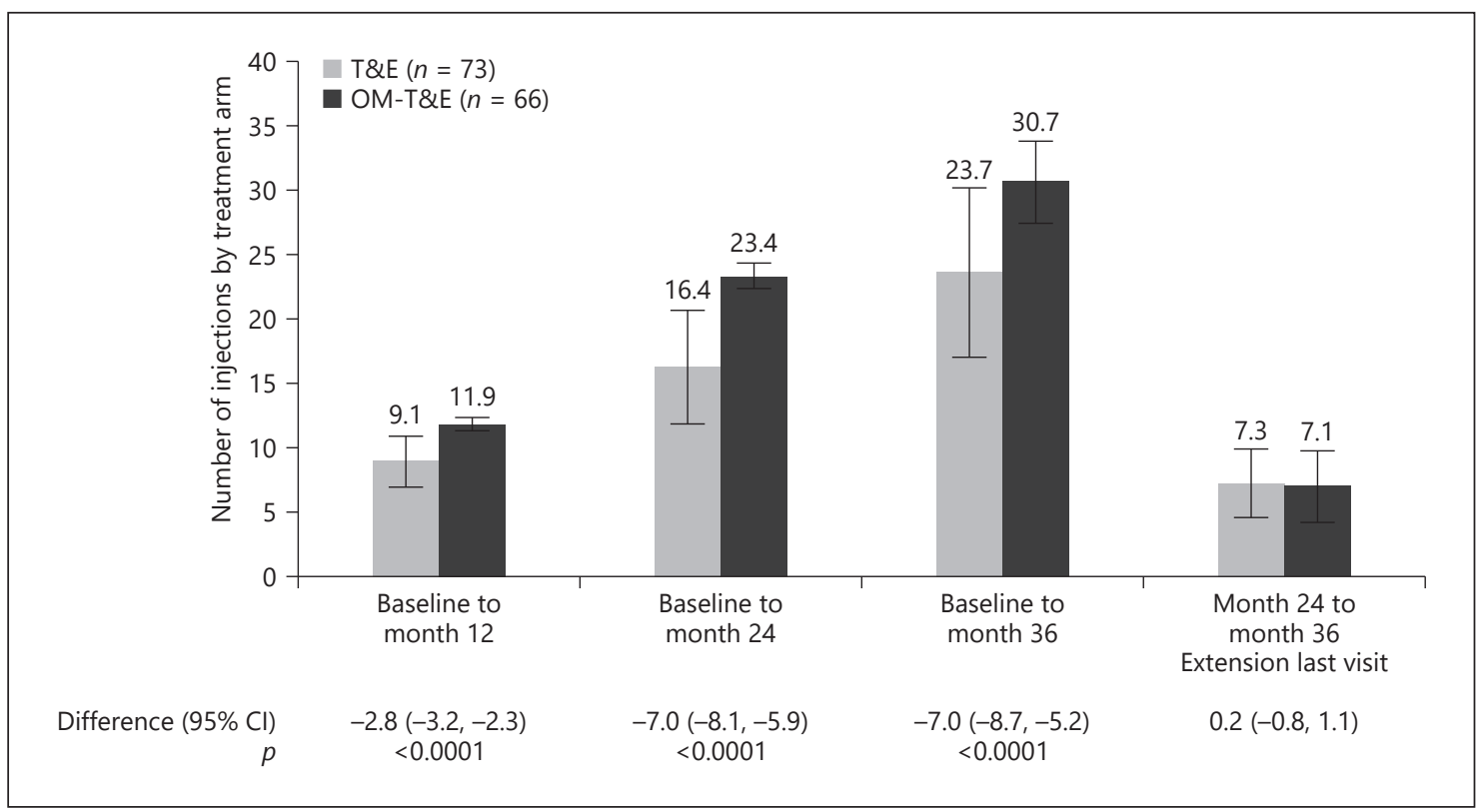

Fig. 3. Mean number of injections during all study phases. Data for the Extension Last Visit cohorts are taken from the last visits for the subjects in the study within a window of months 33-36. CI, confidence interval; $n$, number of patients with data.

The mean (SD; 95\% CI) numbers of injections administered during the extension were $7.3(2.7 ; 6.7-7.9)$ in the $\mathrm{T} \& \mathrm{E}$ group and $7.1(2.8 ; 6.4-7.8)$ in the OM-T\&E group, a difference of $0.2(2.8 ;-0.8$ to 1.1$)$ (Fig. 3). Mean (SD; $95 \% \mathrm{CI}$ ) total injections over the 36-month study period for the patients who participated in the extension were higher in the OM-T\&E group compared with the T\&E group: 30.7 (3.2; 29.9-31.5) versus $23.7(6.6 ; 22.2-25.3)$. The difference in mean injections (95\% CI) from baseline to 36 months was the same as that from baseline to 24 months: -7.0 ( -8.7 to -5.2$)$ versus $-7.0(-8.1$ to -5.9$)$. The most commonly administered number of injections during the extension was 6 (20.9\%) for the overall population, and was $5(26.0 \%)$ and $6(30.3 \%)$ for the T\&E and OM-T\&E groups, respectively (Fig. 4). The median number of injections was 7; however, there was a wide distribution in mean injections: $10.1 \%$ of patients received $1-4$ injections, including $12.1 \%$ of patients in the OM-T\&E 
Fig. 4. Number of injections from month 24 to month 36 by treatment Arm. n, number of patients with data.

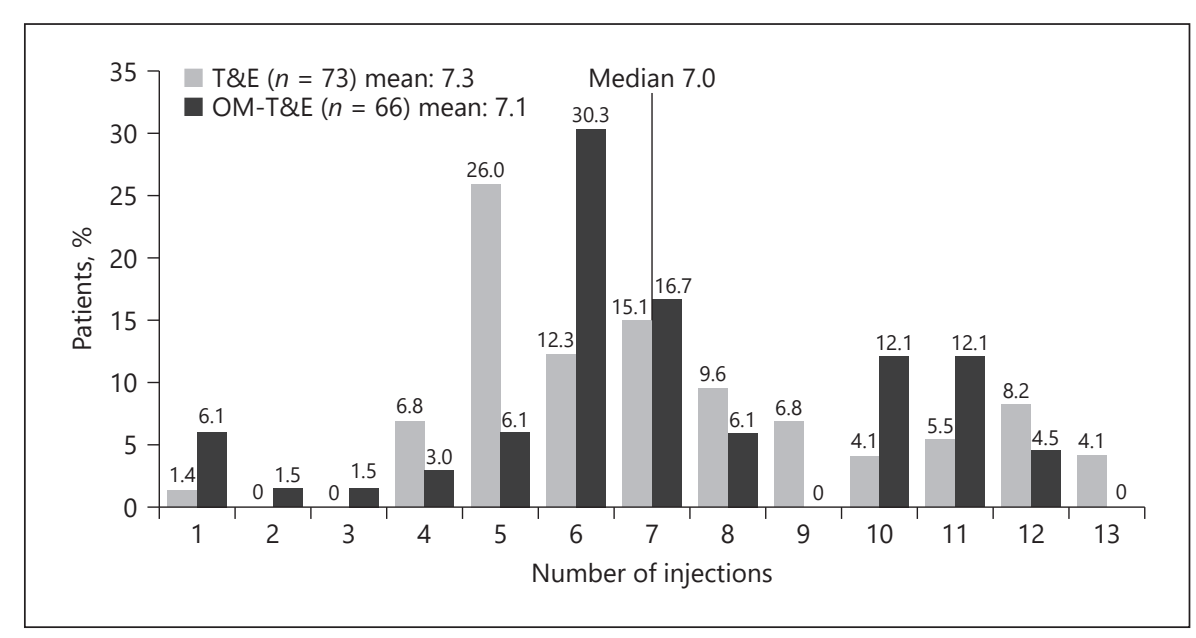

group and $8.2 \%$ in the T\&E group, and $25.2 \%$ of patients received $10-13$ injections, including $28.8 \%$ and $21.9 \%$ in the OM-T\&E and T\&E groups, respectively. A maximum treatment interval of 12 weeks was achieved by $47.9 \%$ of $\mathrm{T} \& \mathrm{E}$ patients and $48.5 \%$ of OM-T\&E patients, while $16.4 \%$ of T\&E patients and $15.2 \%$ of OM-T\&E patients achieved a maximum interval of $<6$ weeks. In their last interval, $30.1 \%$ of T\&E patients and $36.4 \%$ of OM-T\&E patients were at 12 weeks, and $34.2 \%$ of T\&E patients and $39.4 \%$ of OM-T\&E patients were at an interval of $<6$ weeks.

With respect to markers of disease instability, mean (SD) CRT at the patients' last extension visit was 249.3 (58.6) $\mu \mathrm{m}$ in the T\&E group $(n=71)$ and $243.3(55.5) \mu \mathrm{m}$ in the OM-T\&E group $(n=57)$, representing decreases from baseline of -120.1 (113.9) $\mu \mathrm{m}$ and -118.0 (113.5) $\mu \mathrm{m}$, respectively. These reductions are similar to the CRT findings at 24 months (online suppl. Fig. S2). The percentages of patients with any macular fluid were also reduced from $100 \%$ at baseline to $28.2 \%(20 / 71)$ in the T\&E group and $29.8 \%(17 / 57)$ in the OM-T\&E group at their last extension visit, and are comparable with month 24 results (online suppl. Fig. S3).

\section{Discussion/Conclusion}

The 12- and 24-month CANTREAT results demonstrated the effectiveness of a $\mathrm{T} \& \mathrm{E}$ ranibizumab regimen and comparative outcomes to an $\mathrm{OM}$ regimen with significantly fewer injections. This 12-month extension shows that these clinical benefits can be maintained up to 36 months, for patients on a continuous T\&E regimen as well as for patients in OM switching to a T\&E regimen.
Our study determined that the gains from baseline to 12 and 24 months were maintained at 36 months in both the group who persisted on a T\&E regimen and the group who were switched after 24 months of OM to T\&E. We also found that improvements in BCVA and proportions of patients with $\geq 15$-, $\geq 10$-, and $\geq 5$-letter gains and losses were comparable between the 2 groups. In particular, there was no appreciable increase in the percentages of patients who experienced the largest losses of vision; compared with our 24-month data [28], the percentages in the T\&E group were lower at the end of the extension for $\geq 15$-letter ( $4.1 \%$ vs. $6.5 \%)$ and $\geq 10$-letter ( $8.2 \%$ vs. $9.5 \%)$ losses. These results provide important information on the sustainable benefit of a T\&E anti-VEGF strategy. They also support the flexibility of initial choice of regimen with the opportunity for a subsequent switch to T\&E. This is further demonstrated with the observation that the OM-T\&E group were able to reduce their injection interval to the same mean number of injections over the 12-month extension (7.1) versus the group that had been on T\&E throughout CANTREAT (7.3). The continued wide distribution of doses required to manage this patient population underlines the individualized nature of treatment and the importance of providing physicians with dosing flexibility to meet the specific needs of their patients.

The effectiveness of T\&E to maintain 24-month results in both groups was also demonstrated with reductions in CRT and macular fluid. From months 24 to 36, the mean CRT measurement was stable, with a $+0.9 \mu \mathrm{m}$ change for the T\&E group and $+1.9 \mu \mathrm{m}$ for the OM-T\&E group. The percentage of T\&E patients with any macular fluid was $2.2 \%$ higher $(26.0 \%-28.2 \%)$ and was $9.5 \%$ higher $(20.3 \%-29.8 \%)$ in the OM-T\&E group. 
Few prospective trials provide long-term ( $\geq 3$ years) follow-up evaluating the effectiveness of a T\&E regimen in the management of nAMD, which is essential given the chronic nature of this disease. BCVA improvements at 36 months are slightly higher in the CANTREAT T\&E subgroup versus the T\&E-only group of TREX-AMD $(n=$ 21 ) at 36 months (+6.6 vs. +5.0 letters from baseline), with fewer mean injections in the final year of study ( 7.3 vs. 9.2) [9]. The SEVEN-UP (7 years) trial and open-label extension to CATT (5 years) demonstrated that neither monthly nor as-needed anti-VEGF dosing maintain BCVA improvement over the long term $[7,8]$. It is postulated that maintaining a low suppression dose with long-term T\&E may not oversuppress VEGF as may occur with monthly dosing and may allow for better VA maintenance, while preventing the adverse potential effects of recurring edema and treatment that may result in association with prn dosing.

In an open-label extension of the VEGF Trap-Eye: Investigation of Efficacy and Safety in Wet AMD study (VIEW), 323 patients with nAMD and a mean BCVA of 65.8 letters received modified quarterly dosing of aflibercept $2 \mathrm{mg}$ from week 96 to week 192 [30]. The mean gain in BCVA from baseline to week 96 in VIEW $1(+10.2$ letters) was maintained at 192 weeks (+7 letters), and the proportions of patients who gained $\geq 15$ letters were $37 \%$ at week 96 and $30 \%$ at week 192. A mean of 9.6 injections was administered during the extension.

Analysis of clinical information from a large prospective international voluntary $\mathrm{nAMD}$ registry found that the mean change in VA during the maintenance phase of T\&E regimens was +1.0 at 12 months and -1.5 at 36 months [31]. The median treatment interval increased from 35 days at study baseline to 60 days at 36 months, and $38.5 \%$ of eyes had inactive disease throughout the maintenance phase (mean 945 days). The investigators determined that treatment intervals $>12$ weeks were associated with increased risk of reactivation.

A retrospective consecutive case series by Rayess et al. [32] found that T\&E administration of either ranibizum$\mathrm{ab}$ or bevacizumab resulted in a mean gain in BCVA of $13.6 \pm 11.4$ letters among the 59 patients who completed 3 years of treatment. Berg et al. [33] conducted a retrospective review of T\&E anti-VEGF therapy of 155 patients with nAMD, 40 of whom completed 8 years of follow-up. Bevacizumab was given to all patients for the first year, patients were switched to ranibizumab for the second year and then switched back to bevacizumab at the third year, with the possibility of switching to ranibizum$a b$ and (at 6 years) aflibercept at the discretion of the treating ophthalmologist. For the subgroup who completed 8 years of follow-up, BCVA was most improved $(+10.1$ letters $)$ at 2 years, was +8.6 letters at 3 years, and gradually declined to -2.1 letters at 8 years. During the first year, the mean (SD) number of injections among this subgroup was 6.1 (2.4), rising to 10.9 (4.6) on ranibizum$\mathrm{ab}$ during the second year, and was 5.4 (3.5) injections during the eighth year.

The results of our 12-month extension to CANTREAT are comparable to these few long-term studies of T\&E in maintaining BCVA despite a low mean number of ranibizumab injections. Our data support the 24-month findings of CANTREAT that, upon reaching clinical stability, the dosing interval can be extended without loss of visual improvements relative to monthly injections. Additionally, patients who have been following an OM regimen can be switched to a T\&E strategy, with associated reduction in the number of injections, without experiencing loss of visual improvement.

Common to T\&E trials, this extension is limited by open-label design and treatment decisions based on individual physician judgment, which may introduce some bias. As an extension, there exists the potential of a selection bias to include patients who have responded favorably to the initial study treatment; however, our comparison data at baseline and 12,24, and 36 months were collected from the same patient subgroup. These results achieved with ranibizumab may not be reproducible with other anti-VEGF agents administered on a $\mathrm{T} \& \mathrm{E}$ regimen with respect to BCVA or number of injections. The strengths of this extension study are the prospective design, good adherence to study protocol, and multicenter enrollment.

In conclusion, in this 12-month extension to the 24-month CANTREAT study, a T\&E ranibizumab regimen was shown to be effective in maintaining 12- and 24-month gains in BCVA in patients who had followed T\&E throughout the previous 24 months as well as those who were switched from OM treatment to T\&E. These results were achieved with fewer injections in both groups compared with OM dosing. This 36-month study contributes important long-term data regarding the benefit of a T\&E anti-VEGF regimen for the management of patients with nAMD.

\section{Acknowledgments}

The authors wish to acknowledge Jeff Alexander, SNELL Medical Communication, for his medical writing assistance, and Joanne Gavalakis for reviewing and supervising the development of the manuscript. 


\section{Statement of Ethics}

HUMAN SUBJECTS: Human subjects were included in this study. The human Ethics Committees approved the study extension (IRB Services, Aurora, ON, Canada; Health Research Ethics Authority, St. John's, Newfoundland [Reference ID 13.124]; UHN Research Ethics Board, Toronto, ON, Canada [Reference ID 136265B]; Hamilton Integrated Research Ethics Board [Reference ID 13-542], Hamilton, ON, Canada; Health Research Ethics Board, Research Ethics Office, Edmonton, AB, Canada [Reference ID pro00039544]; Ottawa Hospital Research Ethics Board, Ottawa, ON, Canada [Reference ID: 20130394-014]; Sunnybrook Research Ethics Board, Sunnybrook HSC, Toronto, ON, Canada [Reference ID: 176-2013]; St. Michael's Hospital Research Ethics Board, Toronto, ON, Canada [Reference ID: 13-276]; Western University Research Ethics Board, Research Western Support Services, London, ON, Canada [Reference ID: 103854]; Research Ethics Review Committee, Alberta Innovates e Health Solutions, Edmonton, AB, Canada [Reference ID: 2800]; Horizon Health Network Research Ethics Services, Saint John Regional Hospital, Saint-John, New Brunswick [Reference ID: 2013-1867]; Capital Health Research Ethics Board, Center for Clinical Research, Halifax, NS, Canada) and all research adhered to the tenets of the Declaration of Helsinki. All participants provided informed consent. No animal subjects were included in this study.

\section{Conflict of Interest Statement}

Dr. Peter Kertes discloses that he has served on advisory boards for Novartis, Alcon, Roche, and Bayer. His institution has received research funding from Allergan, Bayer, Roche, and Novartis and fellowship funding from Novartis and Bayer. He has stock in Arc-
ticDx. Dr. Tom Sheidow discloses that he has served on advisory boards for Novartis, Bayer, and Alcon. His institution has received research grant-in-aid funding from Novartis ( $>2$ years ago) and QLT ( $>5$ years ago). Dr. Geoff Williams discloses that he has been an Investigator for Novartis, Bayer, Alcon, and Allergan trials. He has served on advisory boards for Novartis, Bayer, Alcon, Allergan, and AbbVie, and has stock in ArcticDx. Dr. Mark Greve discloses that he has served on advisory boards for Novartis, Bayer, and Alcon, and he is a director and shareholder for Secure Diagnostic Imaging. Dr. Ivan Galic discloses that he has served on advisory boards for Novartis, Alcon, and Bayer. Mr. Jason Baker is a Medical Advisor at Novartis Pharmaceuticals Canada.

\section{Funding Sources}

This study was funded by Novartis Pharmaceuticals Canada.

\section{Author Contributions}

Peter Kertes, Ivan Galic, Mark Greve, Geoff Williams, Jason Baker, and Tom Sheidow contributed to conception and design, analysis and interpretation, data collection, obtained funding, and overall responsibility.

\section{Data Availability Statement} able.

\section{References}

1 Li JQ, Welchowski T, Schmid M, Mauschitz MM, Holz FG, Finger RP. Prevalence and incidence of age-related macular degeneration in Europe: a systematic review and meta-analysis. Br J Ophthalmol. 2020 Aug;104(8):107784.

2 Wong WL, Su X, Li X, Cheung CM, Klein R, Cheng CY, et al. Global prevalence of age-related macular degeneration and disease burden projection for 2020 and 2040: a systematic review and meta-analysis. Lancet Glob Health. 2014 Feb;2(2):e106-116.

3 National Eye Institute. Age-related macular degeneration (AMD) data and statistics. Available from: https://www.nei.nih.gov/ learn-about-eye-health/resources-forhealth-educators/eye-health-data-and-statistics/age-related-macular-degeneration-amddata-and-statistics.

4 Klein R, Chou CF, Klein BE, Zhang X, Meuer SM, Saaddine JB. Prevalence of age-related macular degeneration in the US population. Arch Ophthalmol. 2011 Jan;129(1):75-80.

5 Klein R, Klein BE, Knudtson MD, Meuer SM, Swift M, Gangnon RE. Fifteen-year cumula- tive incidence of age-related macular degeneration: the Beaver Dam Eye Study. Ophthalmology. 2007 Feb;114(2):253-62.

6 Friedman DS, O'Colmain BJ, Munoz B, Tomany SC, McCarty C, de Jong PT, et al. Prevalence of age-related macular degeneration in the United States. Arch Ophthalmol. 2004 Apr;122(4):564-72.

7 Rofagha S, Bhisitkul RB, Boyer DS, Sadda SR, Zhang K; SEVEN-UP Study Group. Sevenyear outcomes in ranibizumab-treated patients in ANCHOR, MARINA, and HORIZON: a multicenter cohort study (SEVENUP). Ophthalmology. 2013 Nov; 120(11): 2292-9.

8 Comparison of Age-related Macular Degeneration Treatments Trials (CATT) Research Group; Maguire MG, Martin DF, Ying GS, Jaffe GJ, Daniel E, et al. Five-year outcomes with anti-vascular endothelial growth factor treatment of neovascular age-related macular degeneration: the comparison of age-related macular degeneration treatments trials. Ophthalmology. 2016 Aug; 123(8): 1751-61.
9 Wykoff CC, Ou WC, Croft DE, Payne JF, Brown DM, Clark WL, et al. Neovascular agerelated macular degeneration management in the third year: final results from the TREXAMD randomised trial. $\mathrm{Br} \mathrm{J}$ Ophthalmol. 2018 Apr;102(4):460-4.

10 Wolf A, Kampik A. Efficacy of treatment with ranibizumab in patients with wet age-related macular degeneration in routine clinical care: data from the COMPASS health services research. Graefes Arch Clin Exp Ophthalmol. 2014 Apr;252(4):647-55.

11 Boulanger-Scemama E, Querques G, About F, Puche N, Srour M, Mane V, et al. Ranibizumab for exudative age-related macular degeneration: a five year study of adherence to follow-up in a real-life setting. J Fr Ophtalmol. 2015 Sep;38(7):620-7.

12 Schmidt-Erfurth U, Chong V, Loewenstein A, Larsen M, Souied E, Schlingemann R, et al. Guidelines for the management of neovascular age-related macular degeneration by the European Society of Retina Specialists (EURETINA). Br J Ophthalmol. 2014 Sep; 98(9):1144-167.
Treat-and-Extend Ranibizumab in nAMD: CANTREAT Study Extension
Ophthalmologica 2022;245:230-238 DOI: $10.1159 / 000521517$ 
13 Mehtà H, Tufail A, Daien V, Lee AY, Nguyen $\mathrm{V}$, Ozturk M, et al. Real-world outcomes in patients with neovascular age-related macular degeneration treated with intravitreal vascular endothelial growth factor inhibitors. Prog Retin Eye Res. 2018 Jul;65:127-46.

14 Ciulla TA, Hussain RM, Pollack JS, Williams D. Visual acuity outcomes and anti-vascular endothelial growth factor therapy intensity in neovascular age-related macular degeneration patients: a real-world analysis of 49485 eyes. Ophthalmol Retina. 2020 Jan;4(1):1930.

15 Ozkaya A, Alkin Z, Togac M, Ahmet S, Perente I, Taskapili M. Five-year outcomes of ranibizumab in neovascular age-related macular degeneration: real life clinical experience. Korean J Ophthalmol. 2017 Oct;31(5):42430.

16 Holz FG, Tadayoni R, Beatty S, Berger A, Cereda MG, Cortez R, et al. Multi-country real-life experience of anti-vascular endothelial growth factor therapy for wet age-related macular degeneration. Br J Ophthalmol. 2015 Feb;99(2):220-6.

17 American Society of Retina Specialists. ASRS preferences and trends (PAT) membership survey; 2018.

18 Wykoff CC, Clark WL, Nielsen JS, Brill JV, Greene LS, Heggen CL. Optimizing antiVEGF treatment outcomes for patients with neovascular age-related macular degeneration. J Manag Care Spec Pharm. 2018 Feb; 24(2-a Suppl):S3-15.

19 Rufai SR, Almuhtaseb H, Paul RM, Stuart BL, Kendrick T, Lee $\mathrm{H}$, et al. A systematic review to assess the "treat-and-extend" dosing regimen for neovascular age-related macular degeneration using ranibizumab. Eye. 2017 Sep; 31(9):1337-44.
20 Vardarinos A, Gupta N, Janjua R, Iron A, Empeslidis T, Tsaousis KT. 24-month clinical outcomes of a treat-and-extend regimen with ranibizumab for wet age-related macular degeneration in a real life setting. BMC Ophthalmol. 2017 Apr 27;17(1):58.

21 Kim LN, Mehta H, Barthelmes D, Nguyen V, Gillies MC. Metaanalysis of real-world outcomes of intravitreal ranibizumab for the treatment of neovascular age-related macular degeneration. Retina. 2016 Aug;36(8):141831.

22 Agarwal A, RhoadesWR, Hanout M, Soliman MK, Sarwar S, Sadiq MA, et al. Management of neovascular age-related macular degeneration: current state-of-the-art care for optimizing visual outcomes and therapies in development. Clin Ophthalmol. 2015 Jun 5;9:100115.

23 Arnold JJ, Campain A, Barthelmes D, Simpson JM, Guymer RH, Hunyor AP, et al. Twoyear outcomes of "treat and extend" intravitreal therapy for neovascular age-related macular degeneration. Ophthalmology. 2015 Jun; 122(6):1212-9.

24 Stewart MW. Individualized treatment of neovascular age-related macular degeneration: what are patients gaining? Or losing? J Clin Med. 2015 May 21;4(5):1079-101.

25 Writing Committee for the UK Age-Related Macular Degeneration EMR Users Group. The neovascular age-related macular degeneration database: multicenter study of 92,976 ranibizumab injections: report 1: visual acuity. Ophthalmology. 2014 May;121(5):1092101.

26 Spaide R. Ranibizumab according to need: a treatment for age-related macular degeneration. Am J Ophthalmol. 2007 Apr;143(4): $679-80$.
27 Kertes PJ, Galic IJ, Greve M, Williams RG, Rampakakis E, Scarino A, et al. Canadian treat-and-extend analysis trial with ranibizumab in patients with neovascular age-related macular disease: one-year results of the randomized Canadian treat-and-extend analysis trial with ranibizumab study. Ophthalmology. 2019 Jun;126(6):841-8.

28 Kertes PJ, Galic IJ, Greve M, Williams G, Baker J, Lahaie M, et al. Efficacy of a treat-andextend regimen with ranibizumab in patients with neovascular age-related macular disease: a randomized clinical trial. JAMA Ophthalmol. 2020 Mar 1;138(3):244-50.

29 World Medical Association. World medical association declaration of Helsinki: ethical principles for medical research involving human subjects. JAMA. 2013 Nov 27;310(20): 2191-4.

30 Marcus DM. Long-term follow-up of intravitreal injection for neovascular age-related macular degeneration in an open-label extension of the VIEW 1 Study. Abstract presented at the Association for Research in Vision and Ophthalmology (ARVO) Annual Meeting. Orlando; 2014 May. p. 4-8.

31 Essex RW, Nguyen V, Walton R, Arnold JJ, McAllister IL, Guymer RH, et al. Treatment patterns and visual outcomes during the maintenance phase of treat-and-extend therapy for age-related macular degeneration. Ophthalmology. 2016 Nov; 123(11):2393400

32 Rayess N, Houston SK 3rd, Gupta OP, Ho AC, Regillo CD. Treatment outcomes after 3 years in neovascular age-related macular degeneration using a treat-and-extend regimen. Am J Ophthalmol. 2015 Jan;159(1):3-1.

33 Berg K, Roald AB, Navaratnam J, Bragadóttir R. An 8-year follow-up of anti-vascular endothelial growth factor treatment with a treatand-extend modality for neovascular age-related macular degeneration. Acta Ophthalmol. 2017 Dec;95(8):796-802. 